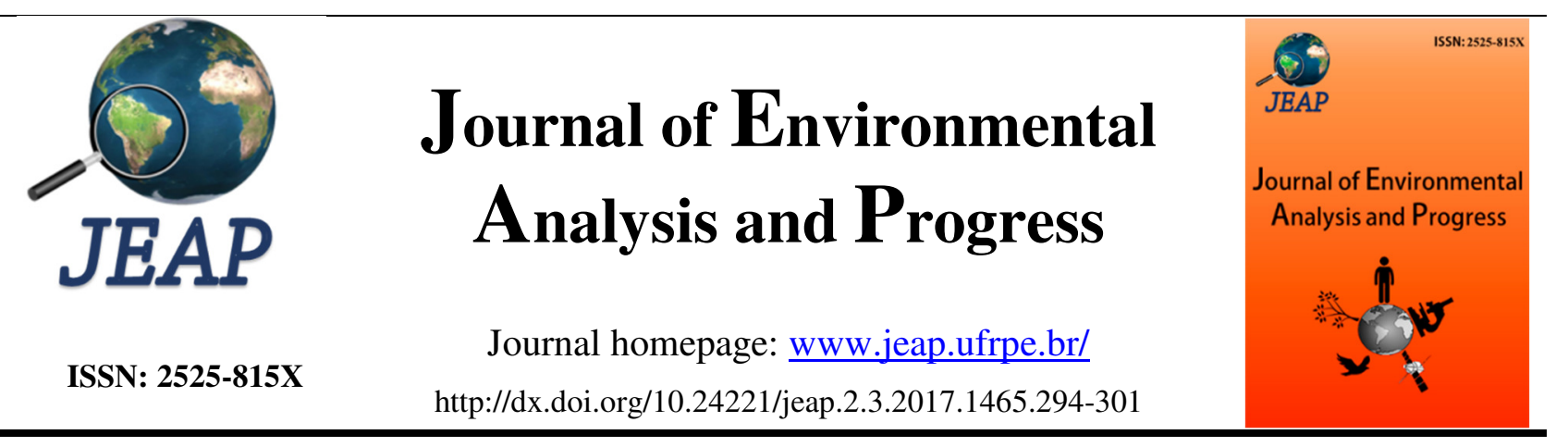

\title{
Umidade volumétrica do solo medida e estimada pelo modelo DSSAT/CROPGRO em cultivo de soja
}

\section{Soil volumetric moisture measured and estimated by the DSSAT/CROPGRO model in soybean cultivation}

Izael Martins Fattori Junior ${ }^{\mathrm{a}}$, Evandro Henrique Figueiredo Moura da Silva ${ }^{\mathrm{a}}$, Alexandre Ortega Gonçalves $^{\mathrm{a}}$, Fábio Ricardo Marin ${ }^{\mathrm{a}}$

a Escola Superior de Agricultura "Luís de Queiros”, Departamento de Engenharia de Biossistemas. Av. Pádua Dias, n. 11 Piracicaba, São Paulo, Brasil. CEP: 13418-900. E-mail: izael.fattori@usp.br; ehfmsilva@usp.br; alexandre.ortega@embrapa.br; fabio.marin@usp.br.

\section{A R T I C L E I N F O}

Recebido 30 Jun 2017

Aceito 27 Jul 2017

Publicado 31 Jul 2017

\begin{abstract}
A B S T R A C T
Agriculture in Brazil has a high-water demand, and is important quantify soil moisture in the management of irrigation and consequently in the optimization of water resources. The measure of this variable is possible through agricultural systems modeling. Aiming to study the dynamics of water in the soil was realized an experiment in the research area of a soy crop. A weather station was installed in the experimental area for the collection of input data for the simulation of the water content through the Soil Water Balance (SWBM), a model of Decision Support System for Agrotechnology Transfer for a generic crop model (DSSAT/CROPGRO). The simulation curve obtained by the modeling was correlated with data measured by the sensor GS3 Decagon in the depths of $0.2 \mathrm{~m}$ e $0.5 \mathrm{~m}$. The inputs and outputs of the system were the precipitation and daily evapotranspiration, by the method of Bowen's Reason. The model showed a medium precision, and good accuracy for both of depths investigated.
\end{abstract}

Keywords: Water dynamics, water balance, evapotranspiration, Bowen ratio.

\section{R E S U M O}

A agricultura no Brasil apresenta uma elevada demanda hídrica, necessitando de pesquisas nesse segmento. Quantificar a umidade do solo tem grande importância no manejo da irrigação e consequentemente na otimização dos recursos hídricos. A mensuração desta variável pode ser realizada através da modelagem de sistemas agrícolas. Objetivando estudar a dinâmica da água no solo realizou-se um experimento em área de pesquisa, em uma lavoura de soja. Uma estação meteorológica foi instalada na área experimental para a coleta de dados de entrada para a simulação do teor de água através do módulo Soil Water Balance (SWBM) do Decision Support System for Agrotechnology Transfer para um modelo genérico de cultivo (DSSAT/CROPGRO). A curva de simulação obtida pela modelagem foi correlacionada com dados medidos pelo sensor GS3 Decagon nas profundidades de $0,2 \mathrm{~m}$ e $0,5 \mathrm{~m}$. As entradas e saídas de água do sistema foram a precipitação e evapotranspiração diária, pelo método da Razão de Bowen. O modelo mostrou uma precisão mediana e boa acurácia para as duas profundidades investigadas.

Palavras-Chave: Dinâmica da água, balanço hídrico, evapotranspiração, razão de Bowen. 


\section{Introdução}

A agricultura é a atividade com maior demanda hídrica no Brasil. Segundo a Associação Brasileira da Indústria de Máquinas e Equipamentos (ABIMAQ, 2016 EMBRAPA, 2016), o país possui uma área agrícola irrigada de 5,623 milhões de hectares, estando entre os dez maiores países com área irrigada no planeta. Desse modo, têm sido necessárias pesquisas que possibilitam quantificar a água no solo e, assim, se ter um manejo hídrico mais eficiente.

Segundo Bernardo et al (2008), o parâmetro umidade do solo está diretamente ligada ao volume armazenado de água, bem como a disponibilidade, compactação entre outros fatores. Com isso é de grande importância o conhecimento deste para que haja a adoção de determinadas práticas de manejo cultural e de irrigação. Sendo assim, uma forma de avaliar a disponibilidade de água no solo é através da simulação realista do balanço hídrico através de modelos.

Os modelos, por definição, são essencialmente teóricos e derivam de um conjunto de teoremas formulados a partir de premissas ou postulados (Alpha \& Chiang, 2005). As teorias são abstrações do mundo real, nesse sentido buscam estreitar problemas complexos (Popper, 2005), sendo, portanto, um recurso encontrado para isolar fatores e relações essenciais de uma complexa realidade: a dinâmica da água no solo, por exemplo.

O Sistema de Suporte à Decisão para Transferência de Agrotecnologia (DSSAT) simula o crescimento, desenvolvimento e produção de uma cultura sob administração prescrita ou simulada, assim como as mudanças de água, solo, carbono e nitrogênio que ocorrem no sistema de corte ao longo do tempo (Jones et al., 2003). No balanço hídrico do solo no modelo CROPGRO, descrito por Ritchie (1985, 1998), o sistema inclui como variáveis de entrada: irrigação e chuva estimula infiltração, escoamento superficial, transpiração da cultura, evaporação do solo, distribuição da captação de água nas camadas do solo e drenagem da água através do perfil.

Dessa forma, o objetivo desse estudo foi caracterizar a dinâmica de água no solo, medida e simulada, comparando a umidade e correlacionando a entrada e saída de água no sistema.

\section{Material e Métodos}

Os dados foram provenientes de um experimento conduzido no município de Piracicaba-SP, na Fazenda Areão na área de pesquisa do Departamento de Engenharia de Biossistemas da Escola Superior de Agricultura
"Luiz de Queiroz" (ESALQ/USP), situado na latitude $22^{\circ} 41^{\prime} 58^{\prime \prime} \mathrm{S}$, longitude $47^{\circ} 38^{\prime} 42^{\prime \prime} \mathrm{O}$ e altitude de $511 \mathrm{~m}$, o clima da região é caracterizado como Cwa, segundo Köppen, em uma área disponível de 3 ha, onde fora delimitada subárea para sua implantação. O tipo de solo é classificado como Argissolo Vermelho Eutrófico (Embrapa, 2006).

A soja (Glycine max L.) Merr) foi plantada no dia 09 de novembro de 2016, adotando-se um sistema de plantio direto em transição antecedido por um consórcio de milho (Zea mays L.) e braquiária (Brachiaria decumbens Stapf. Prain cv. Basilisk), a semeadura obedeceu a um espaçamento de $0,45 \mathrm{~m}$ entre linhas e população de 220 mil plantas por hectare. A cultivar utilizada foi a TMG 7262, cultivar de grupo de maturação 6.2, recomendada para a região, segundo a Tropical Melhoramento \& Genética (TMG, 2017).

Os dados agrometeorológicos foram coletados na própria área experimental, em uma estação de monitoramento ambiental formada por uma torre com os seguintes sensores: dois termohigrômetros (HMP155, Vaisala) com diferença de altura de $0,8 \mathrm{~m}$ entre eles, sendo que o mais baixo ficava localizado $0,2 \mathrm{~m}$ acima do nível do dossel, e acompanhava o crescimento da planta, seguindo a metodologia descrita por Allen et al. (1998). Um pluviômetro (TE525MM, Texas Instruments), com resolução de $0,1 \mathrm{~mm}$, quatro sensores de umidade volumétrica, temperatura e condutividade elétrica do solo (GS3, Decagon), sendo esses instalados na linha e na entrelinha, nas profundidades de $0,2 \mathrm{e}$ $0,5 \mathrm{~m}$, dois sensores de fluxo de calor no solo (HFP01, Hukeseflux), sendo um na linha (G1) e outro na entrelinha $(\mathrm{G} 2)$ e um saldo radiômetro (NR-Lite2, Kipp \& Zone). Os sensores eram conectados a um coletor de dados de 16 canais modelo CR1000 (Campbell Scientific), que fez leituras dos sensores a cada 30 segundo e armazenou os dados a cada 15 minutos.

A saída de água no solo, por meio da evapotranspiração, foi estimada pelo Método da Razão de Bowen (MRB), a partir de medições diretas das temperaturas do bulbo seco e úmido, saldo de radiação e fluxo de calor no solo. Segundo Malek (1993), Azevedo (1999) e Silva (2000), esse método apresenta boa correlação, quando comparado aos valores medidos por lisímetro.

Com as medidas coletadas de saldo de radiação $(\mathrm{Rn})$, fluxo de calor no solo (G1 e G2), diferenças de temperatura $(\Delta \mathrm{T})$ e pressão de vapor $(\Delta \mathrm{e})$ entre os dois níveis acima do dossel da cultura, foi determinado o balanço de energia, conforme a Equação 1:

$R n-G-H-L E=0$ 
onde Rn é o saldo de radiação $\left(M J \cdot m^{-2} \cdot d^{-1}\right), G$ é o fluxo de calor no solo $\left(\mathrm{MJ} \cdot \mathrm{m}^{-2} \cdot \mathrm{d}^{-1}\right)$, H é o fluxo de calor sensível $\left(\mathrm{MJ} \cdot \mathrm{m}^{-2} \cdot \mathrm{d}^{-1}\right)$ e LE é o fluxo de calor latente de evaporação $\left(\mathrm{MJ} \cdot \mathrm{m}^{-2} \cdot \mathrm{d}^{-1}\right)$.

Os valores da razão de Bowen $(\beta)$, foram calculados através da Equação 2 para cada intervalo de 15 minutos, com base nos valores de gradientes de temperatura $(\Delta \mathrm{T})$.

$\beta=\left(\frac{\Delta T u}{(1-w) \Delta T s}-1\right)^{-1}$

onde $\Delta$ Ts é a diferença temperatura do bulbo seco, em ${ }^{\circ} \mathrm{C} ; \Delta \mathrm{Tu}$ é a diferença de temperatura do bulbo úmido, em ${ }^{\circ} \mathrm{C}$; w é o fator de ponderação calculado pelas Equações 3 e 4:

$$
\begin{array}{ll}
\mathrm{W}=0,407+0,0145 * \mathrm{Tu}\left(0^{\circ} \mathrm{C}<\mathrm{T}<16^{\circ} \mathrm{C}\right) & \text { Eq. (3) } \\
\mathrm{W}=0,483+0,01 * \mathrm{Tu}\left(16,1^{\circ} \mathrm{C}<\mathrm{T}<32^{\circ} \mathrm{C}\right) & \text { Eq. (4) }
\end{array}
$$

o fluxo de calor latente será obtido pela Equação (5):

$\mathrm{LE}=\frac{R n-G}{1-\beta}$

Os dados obtidos a cada 15 minutos foram então integrados para serem trabalhados em escala diária. Após serem integrados, os dados foram ajustados para torná-los dados de entrada para o modelo DSSAT/CROPGRO: (a) temperatura máxima do ar, (b) temperatura mínima do ar, (c) saldo de radiação e (d) precipitação pluvial. O módulo utilizado para a simulação da umidade do solo foi o Soil Water Balance (SWBM). Os parâmetros do solo caraterizados de acordo com metodologias proposta por EMBRAPA (1997). Ajustou-se a curva de retenção de umidade no solo pela metodologia proposta por van Genuchten (1980), mediante a utilização do programa (RETention Curve), do Salinity LaboratoryUSDA, segundo Marinho et al. (2016) e inseridos no modelo.

Para se avaliar a eficiência de modelagem, ou seja, os dados observados em função dos dados simulados, foram usados testes estatísticos: (a) coeficiente de determinação, $\mathrm{r}^{2}$ (Equação 6); (b) desvio médio absoluto, DMA (Equação 7); (c) raiz do erro quadrático médio, REQM (Equação 8) e (d) índice de concordância D-Wilmott (Equação 9).

$$
R^{2}=\frac{\sum_{i=1}^{n}\left(E_{i} \quad \bar{O}\right)^{2}}{\sum_{i=1}^{n}\left(\begin{array}{lll}
E_{i} & \bar{O}
\end{array}\right)^{2}+\sum_{i=1}^{n}\left(\begin{array}{ll}
E_{i} & O_{i}
\end{array}\right)^{2}}
$$

onde $\mathrm{R}^{2}$ é o coeficiente de determinação, $O_{i}$ o valor observado, $E_{i}$ o valor simulado e $\bar{O}$ a média dos valores observados.

DMA $=\frac{\sum_{i=1}^{n}\left|e_{t}\right|}{n}$

onde DMA é o desvio médio absoluto, $e_{t}$ a diferença do valor observado e o simulado e $\mathrm{n} o$ número de períodos.

$\mathrm{RQME}=\sqrt{\frac{\sum(\mathrm{Ei}-\mathrm{Oi})^{2}}{\mathrm{n}}}$

onde REQM é a raiz do erro quadrático médio, $\mathrm{O}_{\mathrm{i}}$ o valor observado, $E_{i}$ a média dos valores observados e $n$ o número de períodos.

$$
\mathrm{D}=1-\left[\frac{\sum_{\mathrm{i}=1}^{\mathrm{n}}\left(\mathrm{O}_{\mathrm{i}}-\mathrm{E}_{\mathrm{i}}\right)^{2}}{\sum_{\mathrm{i}=1}^{\mathrm{n}}\left(\left|\mathrm{E}_{\mathrm{i}}+\bar{O}\right|+\left|\mathrm{O}_{\mathrm{i}}-\overline{\mathrm{O}}\right|\right)^{2}}\right]
$$

onde D é o índice de concordância de Willmott (1982), $\mathrm{O}_{\mathrm{i}}$ é o valor observado de ordem $\mathrm{i}, \mathrm{E}_{\mathrm{i}}$ é o valor estimado de ordem i, $\overline{0}$ é a média dos valores medidos, e n o número dos valores medidos.

Ao analisar o código fonte do módulo SWBM do modelo, para calcular o balanço hídrico, foi usada a referência teórica do método do balde unidimensional, descrito por Ritchie $(1985,1998)$, considerando as variáveis de entrada: (a) precipitação cumulativa (RAIN - rain), (b) drenagem diária (DRAIN - Drainage from profile), (c) escorrimento (RUNOF - runoff) e (d) atual número da camada (NLAYR - Actual Number of Soil Layers), conforme a Equação 10.

$\mathrm{TSWINI}=\mathrm{SW}(\mathrm{L}) *$ DLAYR $(\mathrm{L})$

onde TSWINI é a quantidade inicial de água no solo, SW quantidade água no solo em uma determinada camada (L), sendo o DLAYR a espessura dessa camada. 


\section{Resultados}

Todos os dados obtidos, simulados e medidos, passaram por testes para avaliar a competência dos modelos e sua acurácia. Na Tabela 1 são exibidos os valores médios de umidade volumétrica no solo, para as duas profundidades, em conjunto dos resultados estatísticos.

Na Figura 1 observa-se que a profundidade de $0,2 \mathrm{~m}$ mostra o comportamento das curvas de simulação e dos dados medidos próximos, o que condiz com os indicadores de eficiência apresentados. No entanto, nota-se um distanciamento expressivo entre a curva de simulação e os dados mesurados no intervalo entre os DAP (dias após o plantio) 50 e 90. A Figura 2 mostra, através do balanço pluviométrico, uma maior frequência e intensidade de precipitação pluvial no período mencionado. Nesse período, como a umidade de saturação inserida no modelo foi de $0,48 \mathrm{~m}^{3} \cdot \mathrm{m}^{-3}$, o módulo SWBM do DSSAT assumiu, portanto, que o solo atingiu o ponto de saturação. Observa-se também que com o aumento contínuo da precipitação no período mencionado tanto os dados de umidade volumétrica medidos quanto o simulado se mantêm constantes. Pressupõem-se, assim, que o sensor GS3 registrou o ponto de saturação do solo em tal período, com valores próximos de $0,39 \mathrm{~m}^{3} \cdot \mathrm{m}^{-3}$.

Tabela 1. Análise dos dados simulados e obtidos, onde $\bar{\theta}_{\text {DSSAT }}$ é a média da umidade volumétrica simulada e $\overline{\bar{\theta}}_{\text {MEDIDo é a média da umidade volumétrica medida pelo sensor GS3, na Fazenda Areão em Piracicaba-SP. }}$

\begin{tabular}{lcccccc}
\hline Profundidade (m) & $\overline{\boldsymbol{\theta}}_{\text {DSSAT }}$ & $\overline{\boldsymbol{\theta}}_{\text {MEDIDO }}$ & $\mathbf{R}^{\mathbf{2}}$ & DMA & RQME & D \\
\hline 0,2 & 0,397 & 0,359 & 0,571 & 0,047 & 0,084 & 0,876 \\
0,5 & 0,401 & 0,408 & 0,638 & 0,006 & 0,032 & 0,967 \\
\hline
\end{tabular}

\section{Umidade Volumétrica do solo $(20 \mathrm{~cm})$}

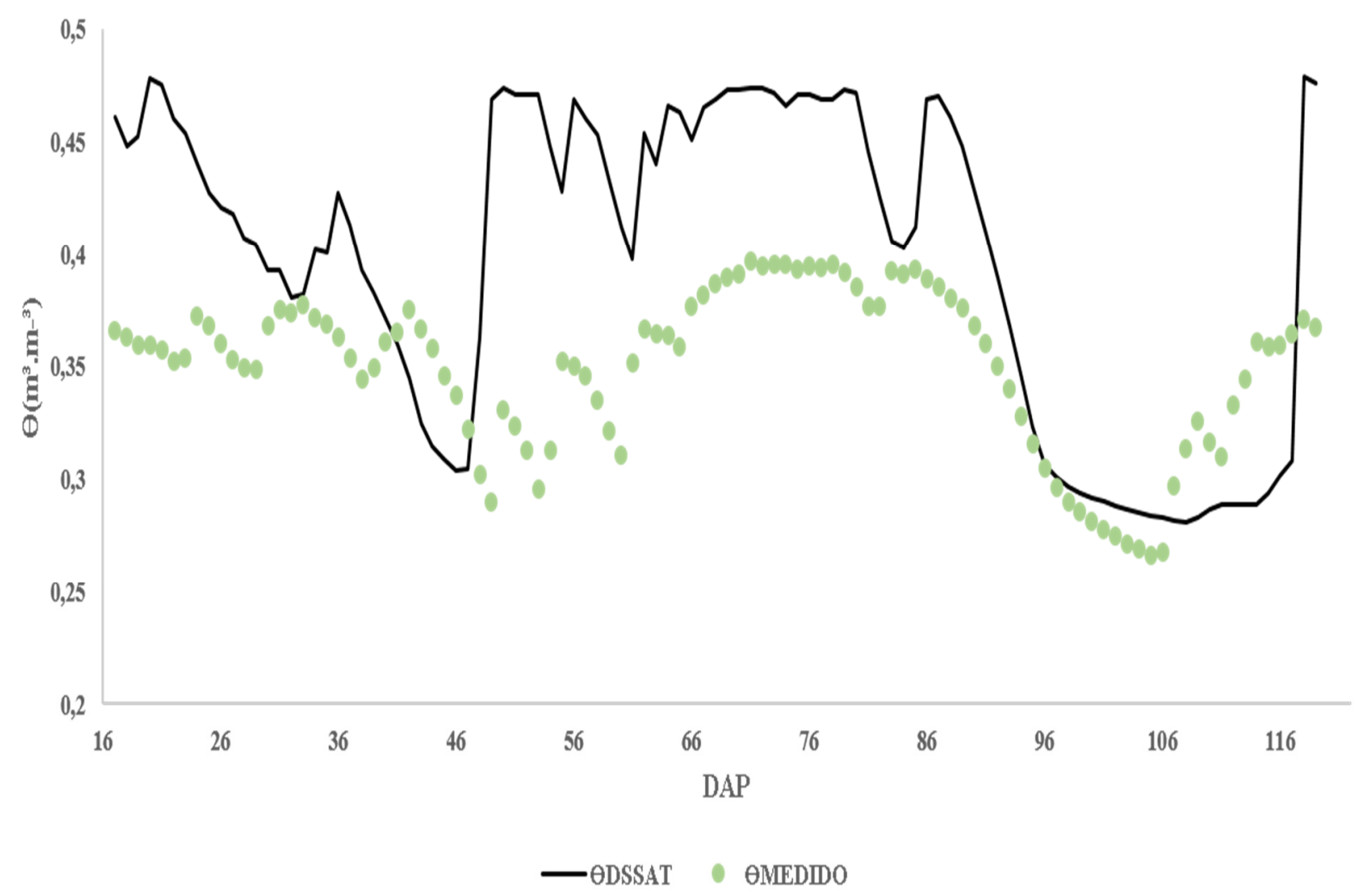

Figura 1 Curva da umidade volumétrica do solo simulada pelo SWBM/DSSAT e valores medidos pelo sensor GS3, na profundidade de $0,2 \mathrm{~m}$.

Comportamento semelhante foi constatado na profundidade de $0,5 \mathrm{~m}$ (Figura 3), porém com melhores resultados de acurácia e precisão. Observa-se, na referida figura, uma boa correlação ao longo do ciclo e uma grande correlação entre os dados medidos e simulados no período DAP 89 e 115. Esse período (Figura 2) mostra altas taxas de evapotranspiração e sem ocorrência de 
precipitação pluvial, indicando perda de água do solo. Na Figura 3 comprova-se uma tendência do modelo para subestimar a umidade volumétrica do solo, em relação aos dados medidos pelo sensor, no intervalo DAP 29 e 48.

Balanço Pluviométrico e Evapotranspiração da Cultura da Soja

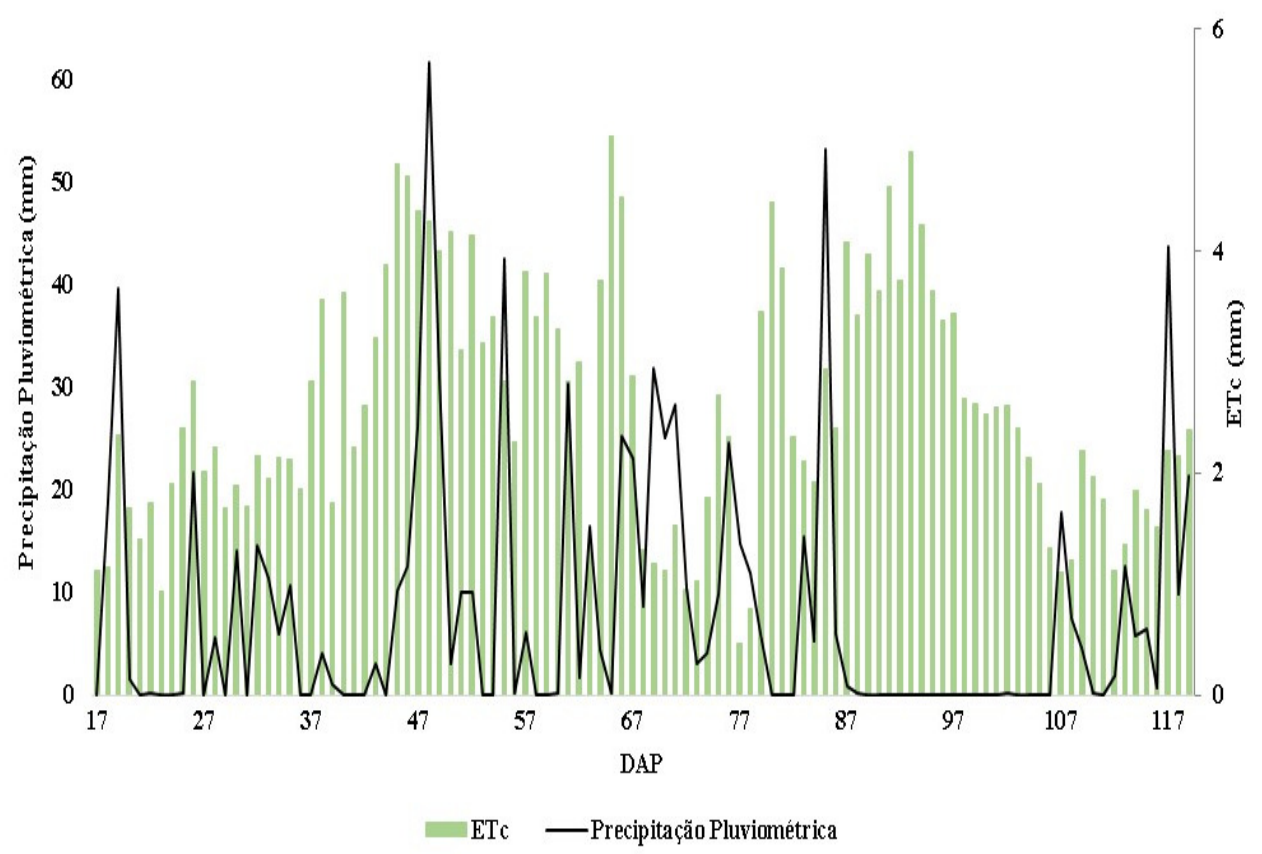

Figura 2 Variação na precipitação pluvial e evapotranspiração diárias.

Umidade Volumétrica do Solo $(50 \mathrm{~cm})$

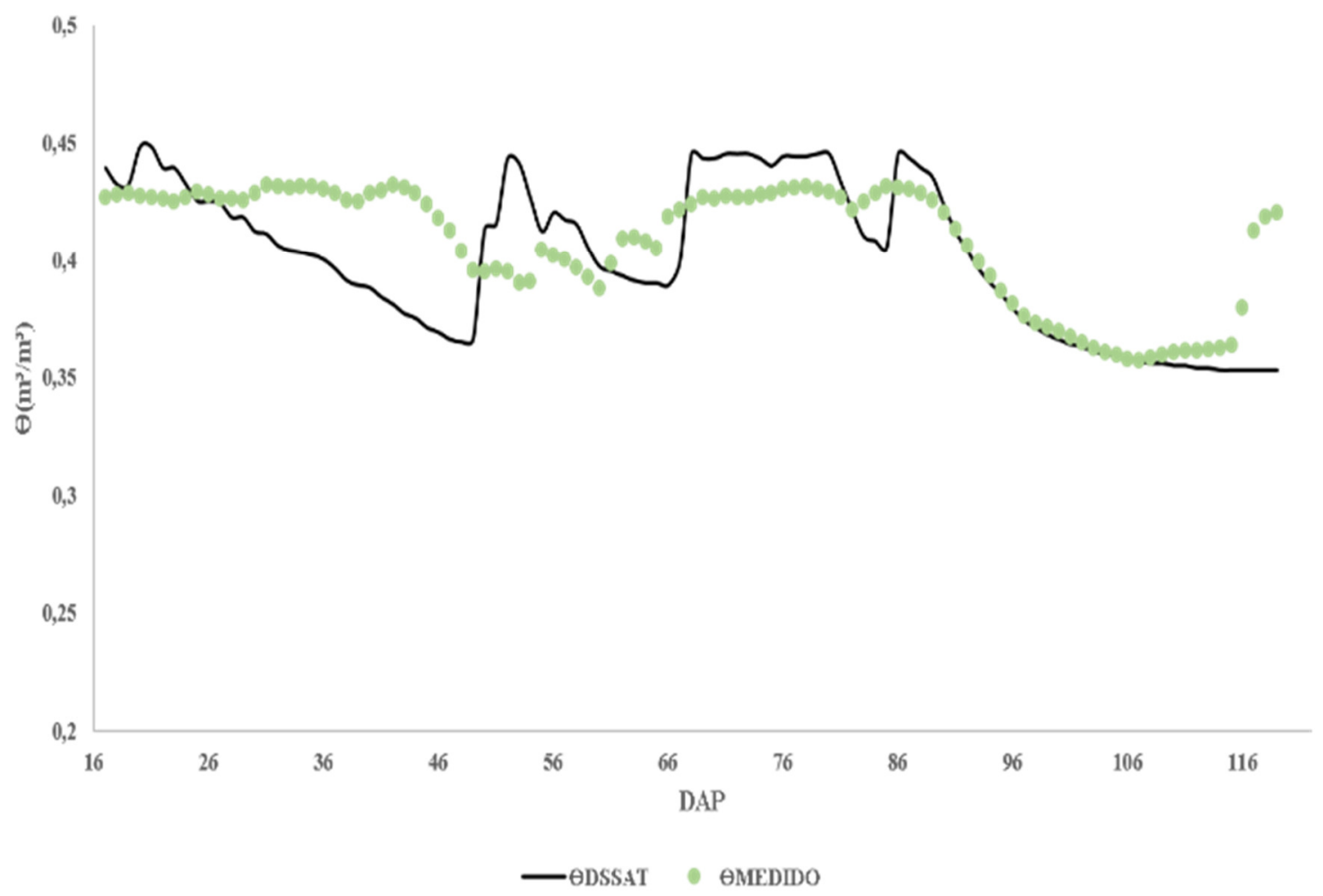

Figura 3 Curva da umidade volumétrica do solo simulada pelo SWBM/DSSAT e valores medidos pelo sensor GS3, na profundidade de $0,5 \mathrm{~m}$.

A Figura 4 mostra a aproximação dos pontos na linha 1:1, para as duas profundidades estudadas. Os testes estatísticos e os indicadores de acurácia e precisão mostraram um melhor desempenho da modelagem para a profundidade de $0,5 \mathrm{~m}$. 

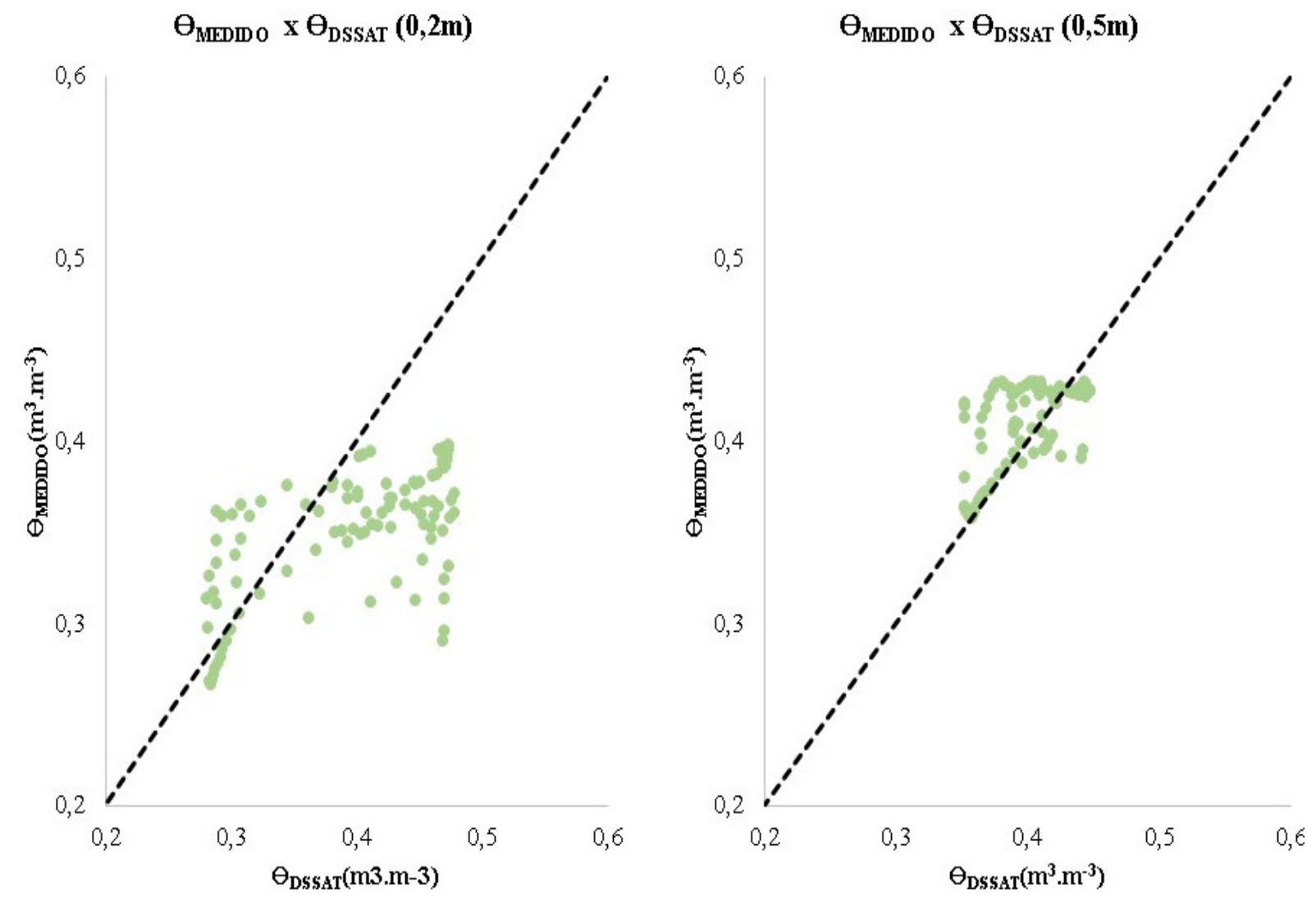

Figura 4 Variação da umidade volumétrica simulada pelo DSSAT em relação à umidade volumétrica medida pelo sensor GS3, na Fazenda Areão em Piracicaba-SP.

\section{Discussão}

Foi encontrada uma precisão mediana do modelo, $\mathrm{R}^{2}$ de, aproximadamente, 0,6 para as duas profundidades do solo, uma vez que a simulação consegue explicar apenas $60 \%$ dos dados medidos pelo sensor.

O teste do DMA apresentou uma boa correlação entre os dados observados e os simulados, esse indicador estatístico pode ser utilizado como uma medida de acurácia do modelo (Wilmott, 1982; Mentzer \& Bienstock, 1998). O mesmo comportamento também foi observado para os indicadores estatísticos RQME e D, indicando boa acurácia do modelo para as duas profundidades de solo. Essa relação também pode ser observada através das duas profundidades estudadas. Tanto os testes estatísticos, quanto os indicadores de acurácia e precisão, mostraram um melhor desempenho da modelagem para a profundidade de $0,5 \mathrm{~m}$.

O sensor GS3, utiliza a técnica da reflectometria no domínio do tempo, conhecida como TDR, uma medida indireta de umidade volumétrica de solos que consiste na emissão de um pulso eletromagnético, cuja velocidade de propagação no meio poroso é função de uma constante denominada constante dielétrica ou permissividade relativa (Righes et al., 2003) O valor da constante dielétrica do solo depende dos teores dos componentes do material poroso (como, agua, ar e minerais) e, nas frequências entre $1 \mathrm{MHz}$ e $1 \mathrm{GHz}$, é muito dependente da umidade volumétrica do solo (Topp et al., 1980). Quanto maior o teor de umidade volumétrica do solo, maior será a constante dielétrica do mesmo e menor será a velocidade de propagação da radiação eletromagnética nele. Como a constante dielétrica é correlacionada com o teor de água do solo, tornase possível estabelecer uma função de relação entre ambas (Rodrigues et al., 2001), de forma que se possa, a partir de uma, determinar-se a outra através de calibrações.

\section{Conclusão}

O módulo SWBM do modelo DSSAT/CROPGRO conseguiu explicar aproximadamente $60 \%$ dos valores de umidade volumétrica do solo, para as profundidades de 0,2 $\mathrm{m}$ e $0,5 \mathrm{~m}$, medidos pelo sensor GS3.

A simulação do teor de umidade volumétrica do solo pelo módulo SWBM do DSSAT apresentou com uma boa acurácia. Foi observada uma tendência de superestimativa do modelo em relação aos dados medidos pelo sensor em alguns intervalos da simulação. No entanto, o 
modelo se mostrou uma boa ferramenta para a simulação do teor de umidade volumétrica do solo.

\section{Agradecimentos}

Ao Grupo de Pesquisa e Experimentação em Modelagem Agrícola (GEPEMA), por todo apoio e conhecimento e ao Departamento de Engenharia de Biossistemas da ESALQ/USP pela infraestrutura para realização desta pesquisa.

\section{Referências}

ABIMAQ. 2016. Atualização da área irrigada no Brasil. Sao Paulo, 2p. Disponível em: http://www.agr.feis.unesp.br/pdf/csei_area_irrigad a_brasil.pdf. Acesso em: 20 abr. 2017.

ALPHA, C. C.; CHIANG, A. C. 2005. Matemática para economistas. São Paulo. Pearson Makron Books.

AZEVEDO, B. M. 1999. Evapotranspiração de referência obtida com a razão de Bowen, lisímetro de pesagem e equação de Penman-Monteith utilizando sistemas automáticos. Tese de Doutorado, Escola Superior de Agricultura "Luiz de Queiroz", Universidade de São Paulo, Piracicaba, 81p.

BERNARDO, S.; SOARES, A. A.; MANTOVANI, E. C. 2008. Manual de irrigação. Universidade Federal de Viçosa. $8^{\mathrm{a}}$ Edição.

DECAGON DEVICES. 2016. GS3 Water content, EC Temperature Sensor: Operator's Manual. Pullman: Decagon Devices, 2016. 27p. Disponível em:

http://manuals.decagon.com/Manuals/13822_GS3

_Web.pdf. Acesso em: 11 maio 2017.

EMBRAPA. 1997. Manual De Métodos de Análise de Solo. Centro Nacional de Pesquisa de Solos. Rio de Janeiro. Segunda Edição.

EMBRAPA. 2006. Sistema Brasileiro de Classificação de Solos. Centro Nacional de Pesquisa de Solos. Rio de Janeiro. Segunda edição.

EMBRAPA. Brasil está entre os países com maior área irrigada do mundo. Disponível em: https://www.embrapa.br/busca-de-noticias//noticia/12990229/brasil-esta-entre-os-paisescom-maior-area-irrigada-do-mundo. Acesso em: 20 mar. 2017.

FARIA, R. T. D.; BOWEN, W. T. 2003. Evaluation of DSSAT soil-water balance module under cropped and bare soil conditions. Brazilian
Archives of Biology and Technology, v. 46, n. 4, p. 489-498.

JONES, J. W.; HOOGENBOOM, G.; PORTER, C. H.; BOOTE, K. J.; BATCHELOR, W. D.; HUNT, L. A.; WILKENS, P. W.; SINGH, U.; GIJSMAN, A. J.; RITCHIE, J. T. 2003. The DSSAT cropping system model. European Journal of Agronomy, v. 18, n. 3, p. 235-265.

MALEK, E. 1993. Rapid changes of the surface soil heat flux and its effects on the estimation of evapotranspiration. Journal of Hydrology, v. 142, n. 1, p. 89-97.

MARINHO, L. B.; FRIZZONE, J. A.; TOLENTINO JÚNIOR, J. B.; PAULINO, J.; FLUMIGAN, D. L.; GÓES, D. B. 2016. Dinâmica da água no sistema solo-planta no cultivo da pimenta tabasco sob déficit hídrico. Irriga: Edição Especial - Irrigação, v. 1, n. 1, p. 246-261.

MENTZER, J. T.; BIENSTOCK, C. C. 1998. Sales forecasting management: understanding the techniques, systems and management of the sales forecasting process. SAGE Publications, Incorporated, ISBN 0761908234.

POPPER, K. 2005. The logic of scientific discovery. Routledge ISBN 1134470029.

RIGHES, A. A.; AMARAL, L. G. H. DO; COSTA, R. D.; ROSA, G. M. DA; WILLES, J. A.; GOMES, A. C. DOS S. 2003. Determinação da água no solo e na planta para irrigação. Santa Maria: Imprensa Universitária. 97p.

RITCHIE, J. T. 1985. A user-orientated model of the soil water balance in wheat. In: Wheat growth and modelling. Springer US. pp. 293-305.

RITCHIE, J. T. 1998. Soil water balance and plant water stress. In: Understanding options for agricultural production. Springer Netherlands. pp. 41-54.

RODRIGUES, T. R. I.; BATISTA, H. S.; CARVALHO, J. M. DE; GONÇALVES, A. O.; MATSURA, E. E. 2001. Uniformidade de distribuição de água em pivô central, com utilização da técnica TDR na superfície e no interior do solo. Revista Brasileira de Engenharia Agrícola e Ambiental, v. 5, n. 2, p. 187-191.

SILVA, F. C. 2000. Determinação da evapotranspiração utilizando o método do balanço de energia e lisímetro de pesagem. Tese de 
Doutorado, Escola Superior de Agricultura "Luiz de Queiroz", Universidade de São Paulo, Piracicaba, São Paulo. 81p.

TMG. Tropical Melhoramento \& Genética. Cultivares de soja sul do Brasil. Disponível em: http://www.tmg.agr.br/cultivar/tmg-7262-rr.

Acesso em: 20 mar. 2017.

TOPP, G. C.; DAVIS, J. L.; ANNAN A. P. 1980. Eletromagnetic determination of soil water content: measurements in coaxial transmission line. Water Resource Research, v. 16, n. 3, p. 574582.

VAN GENUCHTEN, M. T. VAN. 1980. A closed form equation for predicting the hydraulic conductivity of unsaturated soils. Soil Science Society of America Journal, v. 44, p. 892-898.

WILLMOTT, C. J. 1982. Some comments on the evaluation of model performance. Bulletin of the American Meteorological Society, v. 63, n. 11, p. 1309-1313. 